\title{
Design and Fabrication of a Minimally Invasive Surgical Device with Customized Shape Memory Alloy Spring Actuator
}

\author{
Uditha Roshan ${ }^{1, *}$, Ranjith Amarasinghe ${ }^{1}$, Nuwan Dayananda ${ }^{2}$ \\ ${ }^{1}$ Department of Mechanical Engineering, University of Moratuwa, Katubedda 10400, Sri Lanka \\ ${ }^{2}$ Department of Electronic and Telecommunication Engineering, University of Moratuwa, Katubedda 10400, Sri Lanka
}

\section{ARTICLE INFO}

\section{Article History}

Received 15 July 2018

Accepted 11 November 2018

\section{Keywords}

Shape memory alloy

minimally invasive surgeries

gripper

differential scanning calorimetry

\begin{abstract}
A shape memory alloy (SMA)-based spring actuated gripper (SAG) was designed and developed for facilitating minimally invasive surgeries (MIS). Specifically the research consists of design and development of a SMA spring which acts as the actuator for the gripper. A novel mechanism was also developed to transfer the actuator force to the gripper jaws. Further, the actuator force was characterized by a testing apparatus which uses a full-bridge load cell as the force sensing element. The activation temperature of the SMA actuator was obtained by performing differential scanning calorimetry (DSC) tests on the samples.
\end{abstract}

(c) 2018 The Authors. Published by Atlantis Press SARL. This is an open access article under the CC BY-NC license (http://creativecommons.org/licenses/by-nc/4.0/).

\section{INTRODUCTION}

Minimally invasive surgery (MIS) is an emerging and highly favorable surgery procedure compared to open surgeries. MIS has been rapidly developed since late 1980s and it was widely accepted due to several advantages over conventional surgeries [1]. The equipment used to perform MIS are ranging from simple to high-end remotecontrolled devices with the capability to observe the surgical field [2]. The surgical manipulations are performed in a confined space and therefore miniaturization of actuators in such tools is a necessity. For this there are several approaches with each one having their own advantages as well as disadvantages [3]. Among those, using shape memory alloy (SMA) as an actuation material can be considered an attractive approach [4]. The best known SMA for biomedical applications is called NiTiNOL which has several advantages [5].

Over the years several research groups have developed gripper prototypes which are actuated by SMA actuators [6] with different techniques. According to the literature, in most cases Joule heating was used as the activation method of SMA material. However, in this research [7], the SMA material was activated by a heated fluid flow. Temperature and flow rate of the fluid were controlled corresponding to the activation requirements. In addition, a compact mechanism was designed to transfer the force generated by the actuator to the gripping jaws. The kinematics of the mechanism was analyzed to justify the motions. This is highly important for the enhanced performance of the SMA-based spring actuated gripper (SMASAG). The SMA actuator was developed as a spring to get suitable stroke and force. Developed spring type actuator was characterized by using a specially developed force measuring

*Corresponding author. Email: tauroshan@gmail.com apparatus which uses a full-bridge load cell. Furthermore, testing was conducted to find the transformation temperatures of the NiTiNOL material by differential scanning calorimetry (DSC). The actuator mechanism was developed by simple manufacturing techniques and $3 \mathrm{D}$ printing was used to develop the gripper jaws and the enclosure of the mechanism.

\section{DESIGN OF THE SMASAG AND WORKING PRINCIPLE}

\subsection{Working Principle of the SMASAG}

Proposed gripper was constructed by integrating several components. The functionality and importance of each will be described in this section.

The gripper was actuated by a linear actuator structure which was designed as a piston/cylinder assembly (Figure 1). It has an end nut which facilitates easy disassembly. Both the end nut and the piston have mounts to attach the SMA spring inside the cylinder. There are openings in the cylinder body for water to pass for the heating and cooling of the SMA spring. The stroke length of the actuator was designed to be $5 \mathrm{~mm}$.

The linear motion of the piston was converted to an angular motion by the link which is hinged to the base structure. The link consists of two slots that limit the motion and two pins that connect the piston and the driver jaw. The slots were designed in a manner in which no sudden lockups will occur.

The rotational motion of the link is transferred to the driver jaw by the reaction force between the pin and slot joint which makes the jaw 


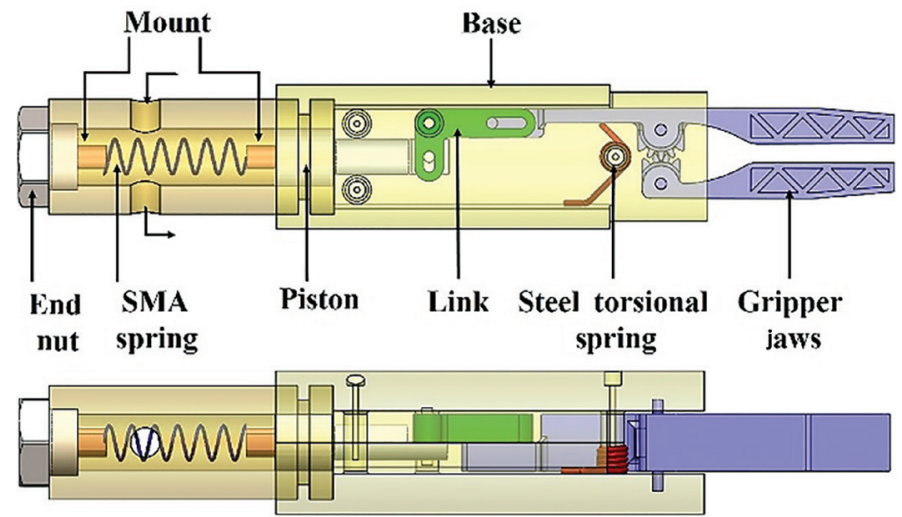

Figure $1 \mid$ Proposed gripper assembly [7]

rotate around a hinge that is located at the Base. In order to compact the mechanism, the gripper pair was connected through a sector gear pair. The main two actuation elements are the SMA spring and the steel torsion spring which support the opening and closing of the gripper jaws respectively. The development and the characterization of the SMA and the torsion spring is elaborated in the following subsections.

\subsection{Design of the SMASAG}

After the designing of the SMASAG, the actuator supporting structure or the outer packaging of the actuator, the SMA based spring and the torsional spring had to be designed.

\subsubsection{Design of the outer packaging of the actuator}

The outer packaging or the structure of the actuator consists of a cylinder which has an outer diameter of $20 \mathrm{~mm}$, a bore of $10 \mathrm{~mm}$ and a length of $50 \mathrm{~mm}$. Also, the piston has a diameter which matches well with the cylinder bore to provide smooth movement and sealing to prevent fluid leakages. Here, the piston is $33.7 \mathrm{~mm}$ in length. The end nut facilitates the final assembly of the actuator. It has $15 \mathrm{~mm}$ across flats and threaded to the cylinder by an M12 standard thread. There are two mounts for the SMA spring attachment and both are threaded to the end nut and piston by M6 standard thread.

\subsubsection{Design of the SMA spring}

The design of the SMA spring is related to the SMA shape setting operation within which a fixture was designed to constrain the spring shape.

According to the design dimensions (in $\mathrm{mm}$ ) in Figure 2, the SMA spring has four turns with $2.24 \mathrm{~mm}$ pitch and the spring diameter is $8 \mathrm{~mm}$.

\subsubsection{Design of the torsion spring}

The torsion spring provides the closing action of the gripper jaws. Having a high spring constant may cause excessive force on the

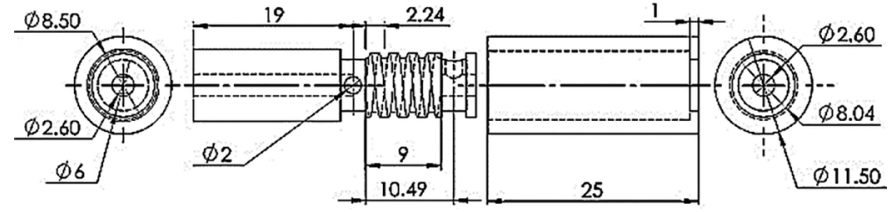

Figure $2 \mid$ The SMA shape setting fixture design

object and partial opening of gripper jaws. On the other hand, having a low spring constant may cause partial gripping. Therefore, the following Eqs. (1)-(10) were used to design the torsional spring with an appropriate spring constant.

$$
\begin{aligned}
F_{\mathrm{LJ}} & =\frac{r_{\mathrm{A}} F_{\mathrm{SMA}} \cos \beta}{r_{\mathrm{B}}} \\
L_{1 x} & =L_{10}-\frac{D_{\mathrm{T}} \theta}{2} \\
C^{2} & =L_{10}^{2}+L_{1 x}^{2}-2 L_{10} L_{1 x} \cos \theta \\
L_{C x} & =L_{C 0}+C \\
F_{\mathrm{JS}} & =\frac{F_{\mathrm{LJ}} L_{\mathrm{H}} \cos (\alpha+\beta)+F_{\mathrm{G}} L_{\mathrm{G}}}{L_{C x}} \\
N_{\mathrm{E}} & =\frac{L_{10}+L_{20}}{3 \pi D_{\mathrm{T}}} \\
N_{\mathrm{A}} & =N_{\mathrm{B}}+N_{\mathrm{E}} \\
k_{\text {per turn }} & =\frac{d_{\mathrm{T}}^{4}}{10.8 D_{\mathrm{T}} N_{\mathrm{A}}} \\
F_{\mathrm{SJ}} & =\frac{d_{\mathrm{T}}^{4}}{10.8 D_{\mathrm{T}} N_{\mathrm{A}}} \frac{\theta}{2 \pi} \\
F_{\mathrm{SJ}} & =F_{\mathrm{JS}}
\end{aligned}
$$

Here $N_{\mathrm{A}}, N_{\mathrm{B}}$ and $N_{\mathrm{E}}$ are the actual, body and effective turns of the torsional spring, respectively.

Figure 3 illustrates the parameters used in the above equations. Equations (1)-(5) were used to calculate the force exerted on the torsional spring due to the actuation force by the SMA spring. Equations (6)-(10) are related to finding the number of turns of the torsion spring.

\section{FABRICATION OF THE SMASAG AND FORCE CHARACTERIZATION OF THE SMA ACTUATOR}

\subsection{Fabrication of the SMA Actuator}

A commercially available SMA material called NiTiNOL was used to fabricate the spring and its composition is $\mathrm{Ni}-55 \% ; \mathrm{H}-0.001 \%$; $\mathrm{O}-0.05 \%$; N - 0.001\%; C - 0.05\%; Ti - Balance. The acquired NiTiNOL material was in the form of straight wires with different diameters and transformation temperatures. Hence, a fixture was required in order to get them in the correct spring shape while conducting shape setting at high temperature. 
As the NiTiNOL wire tends to regain its trained shape when increasing the temperature beyond Austenitic final temperature, it is important to use fixtures (Figure 4) when inducing newly defined shape into the material. Hence, the fixture was placed in a Muffle furnace according to the temperatures and aging times indicated in Table 1. Finally, the sample was quenched in water to obtain the final spring shape (Figure 5).

After shape setting it is important to investigate the SMA characteristic temperatures which are commonly known as transformation temperatures, through a DSC test.

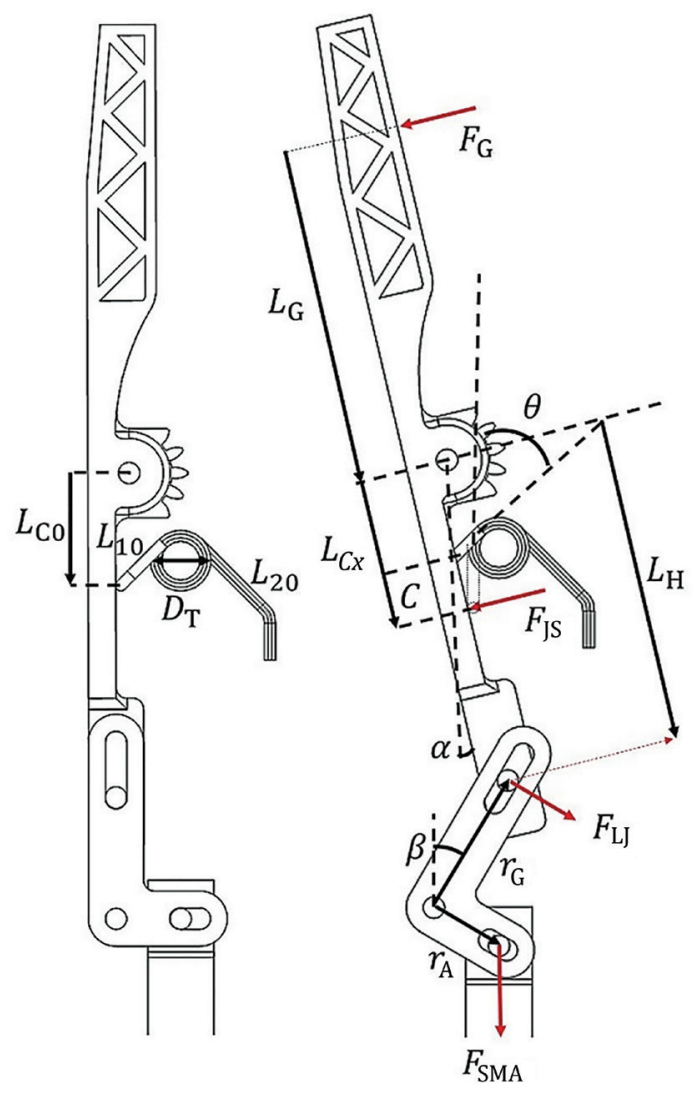

Figure 3 Free body diagram and calculation parameters

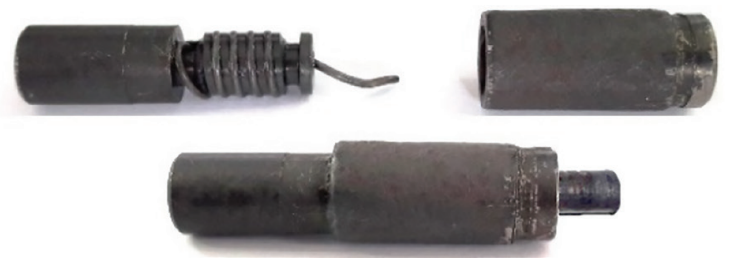

Figure 4 Fabricated fixture

Table 1 NiTiNOL wire shape setting details

\begin{tabular}{lccc}
\hline Sample no. \# & $\begin{array}{c}\text { Wire diameter } \\
(\mathbf{m m})\end{array}$ & $\begin{array}{c}\text { Shape setting } \\
\text { temperature }\left({ }^{\circ} \mathbf{C}\right)\end{array}$ & $\begin{array}{c}\text { Aging time } \\
(\mathbf{m i n})\end{array}$ \\
\hline 1 & 1.0 & 500 & 25 \\
2 & 1.0 & 500 & 30 \\
\hline
\end{tabular}

\subsection{Investigating the Transformation Temperatures of Shape Set Springs}

Differential scanning calorimetric test is primarily based on monitoring the energy changes that are happening in the samples during heating and cooling cycles [8]. These energy changes would either be exothermic or endothermic. By obtaining a heat flow graph with respect to temperature, characteristic temperatures of a SMA sample can be calculated. For this procedure, tests were conducted by DSC Q200 instrument [9]. Tzero aluminum pans were used as the reference and the sample pans. The samples were subjected to a thermal program where they were ramped from 0 to $120^{\circ} \mathrm{C}$ at $2^{\circ} \mathrm{C} / \mathrm{min}$ temperature increments and then cooled from 120 to $0^{\circ} \mathrm{C}$ at $2^{\circ} \mathrm{C} / \mathrm{min}$ temperature decrements. Then the data was stored and plotted as is depicted in the following Figures 6 and 7 and Table 2 summerizes

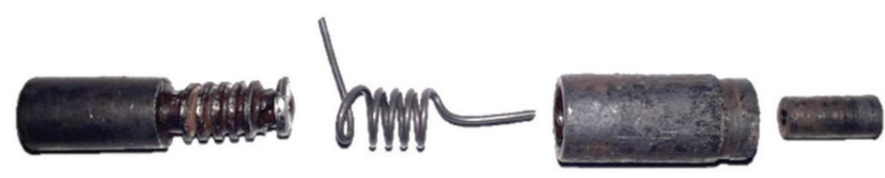

Figure 5 The Shape set NiTiNOL spring

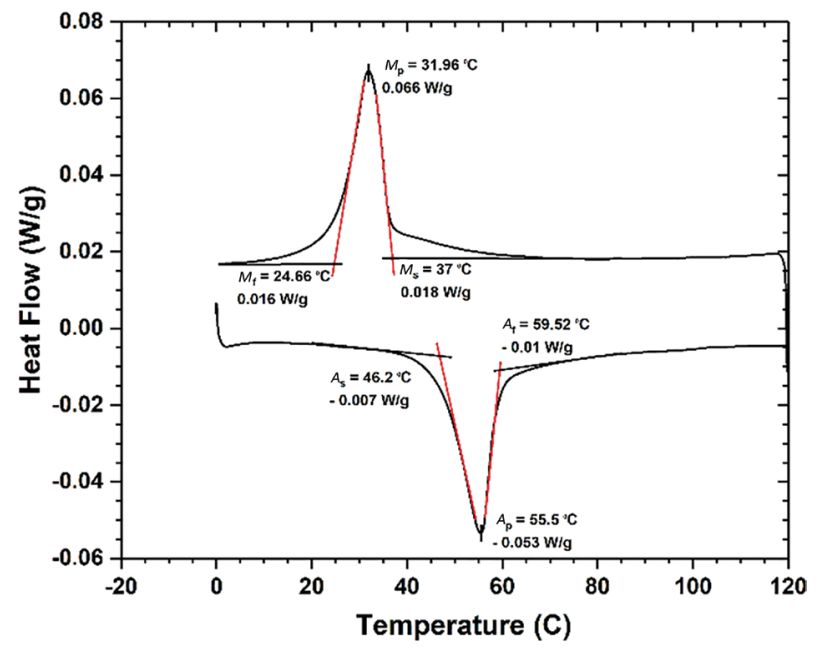

Figure 6 DSC thermogram for sample \#1

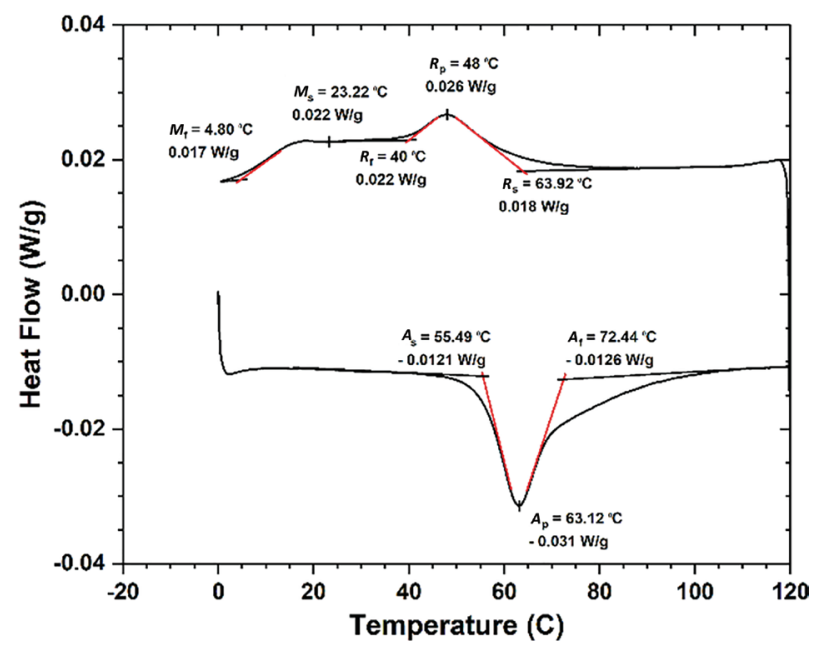

\begin{tabular}{l|l} 
Figure 7 & DSC thermogram for sample \#2
\end{tabular} 
the results observed from the DSC test. $M_{\mathrm{s}}, M_{\mathrm{p}} A_{\mathrm{s}}$ and $A_{\mathrm{f}}$ indicate the Martensite start and finish temperatures and Austenite start and finish temperatures respectively (Table 2).

\subsection{Fabrication of the Outer Packaging and the Gripper}

The fabricated SMASAG is depicted in Figure 8. The base structure and the gripper jaws were made from ABS Plus material with the use of $3 \mathrm{D}$ printing technology.

Actuator piston and cylinder was made from aluminum and brass materials respectively. SMA element mounts were made out of brass and threaded to the piston and End nut which is made out of steel.

All the commonly available materials were used because of achieving easiness of prototyping. When miniaturizing for the actual application more reliable bio-compatible materials need to be used.

Miniaturization of the actuator for real application would make more challenges. Commonly used sensing methods in the macro scale cannot be used in such scenario for temperature measurement. So MEMS based temperature sensing [10] would be more ideal in such situations.

\subsection{Force Characterization of the SMA Spring}

After shape setting the NiTiNOL wire into a spring, it is important to have an understanding about the amount of force that can be exerted through it on the mechanism. Therefore, an apparatus was developed to measure the force that can be achieved when the springs are constrained to recover their initial shape.

The apparatus is shown in Figure 9a. It consists of a full-bridge load cell and a container which was used to immerse the spring in water while one end was rigidly connected to the base of the container

Table 2 Transformation temperatures estimation from DSC test

\begin{tabular}{lccccc}
\hline Sample no. \# & Sample weight $(\mathbf{m g})$ & $\boldsymbol{M}_{\mathrm{s}}\left({ }^{\circ} \mathrm{C}\right)$ & $\boldsymbol{M}_{\mathrm{f}}\left({ }^{\circ} \mathrm{C}\right)$ & $\boldsymbol{A}_{\mathrm{s}}\left({ }^{\circ} \mathrm{C}\right)$ & $\boldsymbol{A}_{\mathrm{f}}\left({ }^{\circ} \mathrm{C}\right)$ \\
\hline 1 & 17.625 & 37 & 24.6 & 46.2 & 59.5 \\
2 & 18.462 & 23.2 & 4.8 & 55.5 & 72.4 \\
\hline
\end{tabular}

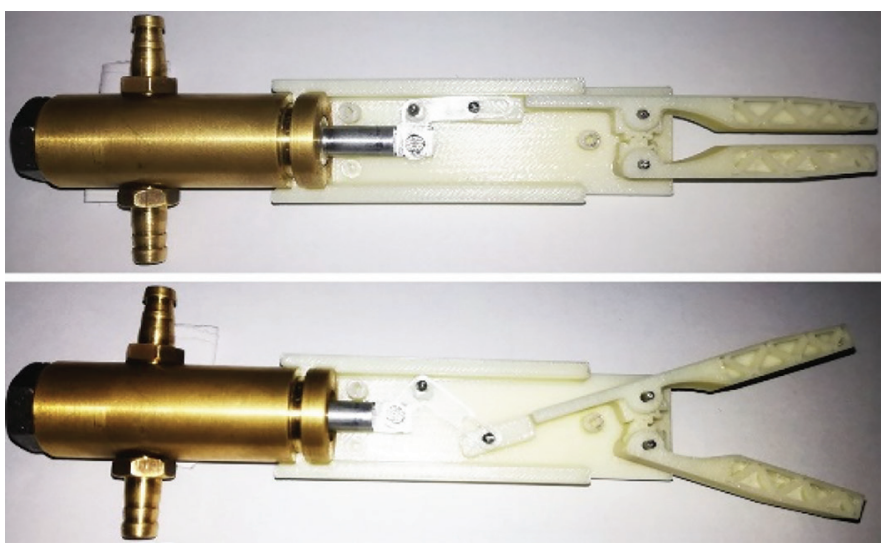

Figure 8 The fabricated SMASAG and the other end spring was attached to measure the force as in Figure 9c. Finally, the container was filled with water and its temperature was gradually increased by a heater. The full-bridge load cell was connected to a 24-bit load cell amplifier (HX711).

The HX711 was connected to a microcontroller (ATmega 2560) based development board in order to acquire data. After obtaining raw data values from the amplifier, the development board was made to transmit the raw data to a computer through serial communication and all the data processing tasks were conducted within a LabVIEW generated program. In addition, a graphical user interface (GUI) was developed to plot and display the data.

Simultaneously the temperature of the water was measured through a four and half true RMS Digital Multimeter which is compatible with thermocouple probes. The acquired values were plotted and Figure 10 shows the variation of force with the temperature for

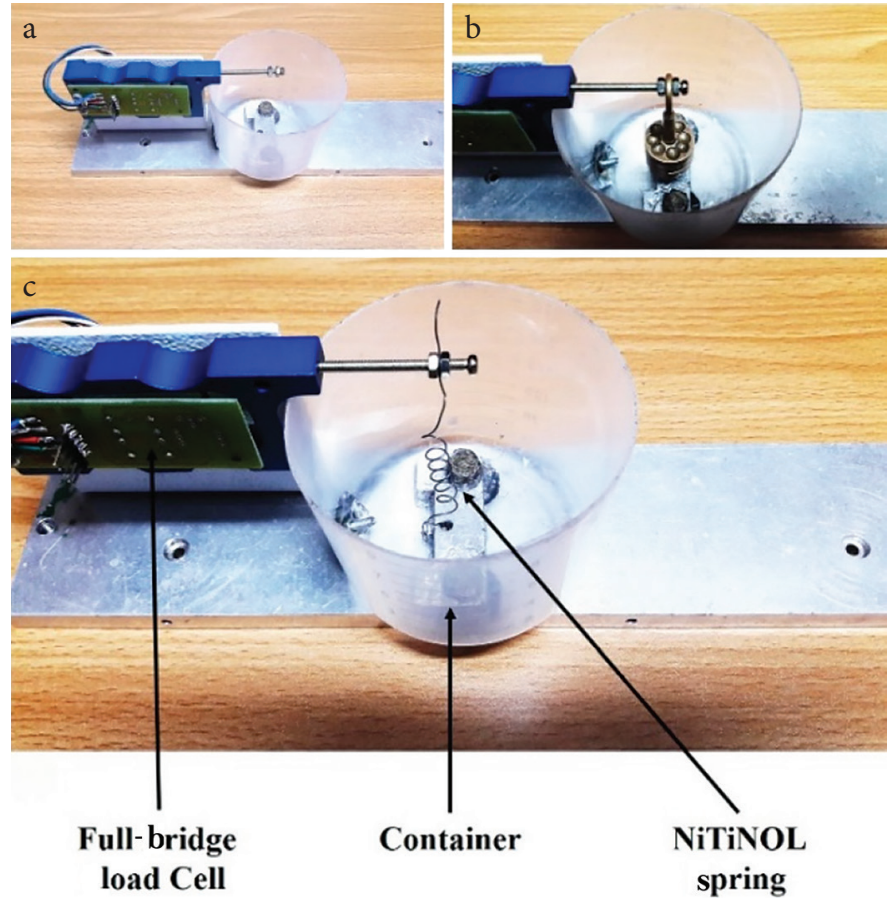

Figure 9 (a) Force measuring apparatus. (b) Calibrating by known weights. (c) NiTiNOL spring attachment

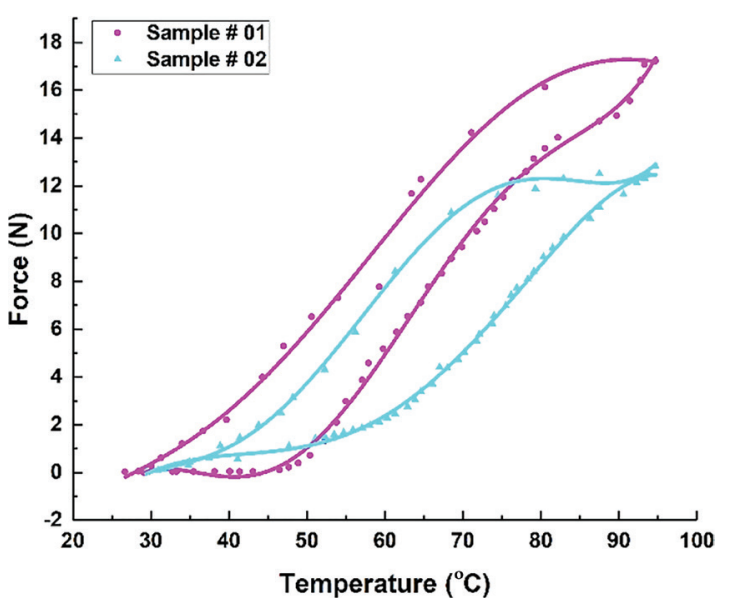

Figure 10 Force characterization results 
the NiTiNOL springs which had the same coil and wire diameter with different transformation temperatures. These forces can be achieved in a controlled manner $[11,12]$.

\section{CONCLUSION}

In this study, a SMA spring actuator-based gripper was designed and fabricated. For this purpose, the tasks carried out included the force characterization of a NiTiNOL spring and development of a novel mechanism to transfer the actuator force to the gripper jaws. The results showed that the required force could be achieved quickly for the springs having lower transformation temperatures if they were subjected to the same conditions. Furthermore, to investigate the transformation temperature of the actuator material, a DSC test was conducted. The test result showed that the Austenite finish $\left(A_{\mathrm{f}}\right)$ temperature had increased with the annealing time.

\section{REFERENCES}

[1] C. Song, History and current situation of shape memory alloys devices for minimally invasive surgery, Open Med. Dev. J. 2 (2010), 24-31.

[2] R.H. Petersen, Video-assisted thoracoscopic thymectomy using 5-mm ports and carbon dioxide insufflation, Ann. Cardiothorac. Surg. 5 (2016), 51-55.

[3] J. Mohd Jani, M. Leary, A. Subic, M.A. Gibson, A review of shape memory alloy research, applications and opportunities, Mater. Des. (1980-2015), 56 (2014), 1078-1113.

\section{Authors Introduction}

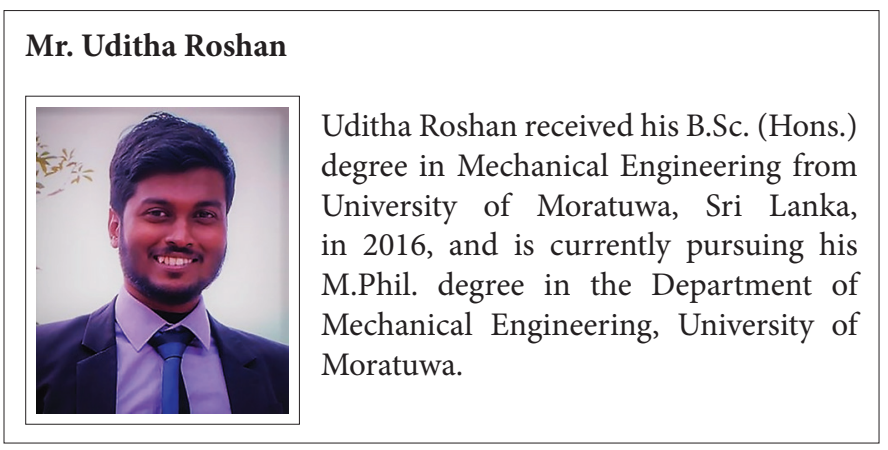

\section{Dr. Nuwan Dayananda}

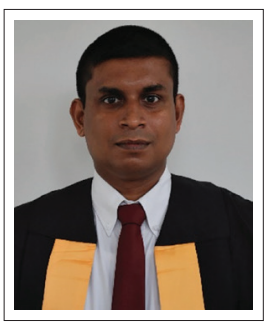

Dr. Nuwan Dayananda is a Senior Lecturer at the Department of Electronic \& Telecommunication Engineering, University of Moratuwa, Sri Lanka. He received his Ph.D. in Biomedical Engineering from Western University, London, Ontario, Canada in 2008. His research is focused on using technology to solve problems in medicine and biology. He is specifically interested in medical imaging and medical image processing related research such as image based diagnosis, image guided surgery and therapy, and surgical simulators.
[4] D. Mantovani, Shape memory alloys: properties and biomedical applications, JOM 52 (2000), 36-44.

[5] D. Stockel, A. Melzer, The use of NiTi alloys for surgical instruments, Conflu. Med. (1995), 790-798.

[6] S. Yan, X. Liu, F. Xu, J. Wang, A gripper actuated by a pair of differential SMA springs, J. Intell. Mater. Syst. Struct. 18 (2007), 459-466.

[7] T.A.U. Roshan, Y.W.R. Amarasinghe, N.W.N. Dayananda, Design and development of a shape memory alloy spring actuated gripper for minimally invasive surgeries, Presented at the International Conference on Artificial Life and Robotics (ICAROB 2018), B-Con Plaza, Beppu, Oita, Japan, 2018, pp. 566-569.

[8] T.G. Bradley, W.A. Brantley, B.M. Culbertson, Differential scanning calorimetry (DSC) analyses of superelastic and nonsuperelastic nickel-titanium orthodontic wires, Am. J. Orthod. Dentofacial. Orthop. 109 (1996), 589-597.

[9] “About TA", TA Instruments. [Online]. Available: https://www.tainstruments.com/about-ta-instruments/. [Accessed: 10-Feb-2018].

[10] B.A.D.J.C.K. Basnayake, Y.W.R. Amarasinghe, R.A. Attalage, A.G.B.P. Jayasekara, M.G.K. Devinda, Development of multisensory feedback system for building automation systems, 2017 IEEE SENSORS, IEEE, Glasgow, UK, 2017, pp. 1-3.

[11] T.A.U. Roshan, B.A.D.J.C.K. Basnayake, Y.W.R. Amarasinghe, D. Wijethunge, N.D. Nanayakkara, Development of a PID based closed loop controller for shape memory alloy actuators, 2018 Moratuwa Engineering Research Conference (MERCon), IEEE, Moratuwa, Sri Lanka, 2018, pp. 460-464.

[12] B.A.D.J.C.K. Basnayake, Y.W.R. Amarasinghe, R.A. Attalage, T.D.I. Udayanga, A.G.B.P. Jayasekara, Artificial intelligence based smart building automation controller for energy efficiency improvements in existing buildings - IJAIST, Int. J. Adv. Inf. Sci. Technol. 4 (2015), 85-91.

\section{Dr. Ranjith Amarasinghe}

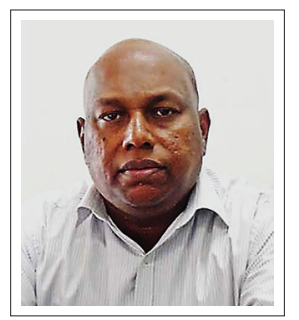

Dr. Ranjith Amarasinghe received his B.Sc. (Hons.) degree in Mechanical Engineering from University of Moratuwa, Sri Lanka, in 2000, his Masters degree in Information Science and Systems Engineering in 2003 and Dr. Eng. degree in Micro Electro Mechanical Systems (MEMS) in 2007 from Ritsumeikan University, Japan. He served as a postdoctoral fellow under Japan Society for the Promotion of Science (JSPS) from 2008 to 2010, and as a Chair Professor from 2010 to 2011 in the Department of Micro Systems Technology, Ritsumeikan University, Japan. From 2011 he joined the University of Moratuwa as a Senior Lecturer. 